\title{
Women with Depression—“You Can't Tell by Looking"TM
}

\author{
Jennifer M. Wilen • Kyle O. Mounts
}

Published online: 3 June 2006

(C) Springer Science+Business Media, Inc. 2006

Keywords Perinatal $\cdot$ Depression $\cdot$ Preconception $\cdot$ Mood disorder

\section{Introduction}

Perinatal depression, a mood disorder that occurs during pregnancy or the first year postpartum, affects $10-15 \%$ of women and up to $28 \%$ of women living in poverty. A previous history of depression, either preconceptionally or during the interconception period, is the strongest predictor that a woman will suffer from perinatal depression. Perinatal depression can adversely affect not only the woman, but also her fetus or infant, and has been linked with prematurity, difficulties with maternal-infant bonding and infant behavior problems later in life. If a woman has a known history of depression, she can be counseled preconceptionally about her increased risk of experiencing a subsequent episode during the perinatal years and thus take an active role in recognizing signs and symptoms of depression and accessing treatment as early as possible should an episode occur. The earlier treatment is sought for perinatal depression, the greater the chance for improved outcomes.

\section{Background}

Depression is currently the leading cause of disease-related disability among women worldwide [1]. Depression can

\section{J. M. Wilen}

Wisconsin Association for Perinatal Care,

K. O. Mounts $(\square)$

Newborn Care Physicians of Southeast Wisconsin,

3070 N51st St., Ste P309, Milwaukee, WI 53210

e-mail:kmounts@pol.net cause moodiness, irritability, changes in sleep patterns, sexual interest or appetite, and an inability to carry on with daily activities. In the most severe cases depression can cause thoughts of harming others or oneself or suicide. Prevalence rates of perinatal depression, which occurs during pregnancy or at some point within the first postpartum year, are similar to those in the general population, affecting $10-15 \%$ of women and up to $28 \%$ of women living in poverty [2,3]. Some studies suggest that a woman's risk of becoming depressed during the early postpartum period is approximately three times that of women in the general population [4]. Depression may affect how a woman is able to relate and respond to her infant and if untreated, may lead to thoughts of harming the infant or infanticide. In addition, depression can have significant effects on prenatal care-seeking behavior [5], substance abuse [6], and infant/child mental health [7-9] and nutrition [10]. Depression has also been associated with low birthweight [11] and premature delivery [12].

Approximately $50 \%$ of women with perinatal depression remain undiagnosed when they are screened based on clinical observations alone [13]. Perhaps the strongest argument for routine screening of all pregnant and postpartum women is the fact that you can't tell simply by looking that a woman is depressed. However, there are some women who may be at increased risk for perinatal depression, including women living in poverty, those in abusive relationships, and women with a prior history of depression [14]. In fact, it is thought that having a history of depression is the strongest predictor that a woman will suffer from an episode of major depression during pregnancy or the postpartum period $[15,16]$. Women with other mental disorders are at risk as well. For example, women with bipolar disorder also have an increased risk of experiencing postpartum depression, and some studies suggest an increased risk for a much more serious illness, postpartum psychosis [17]. Due to the predictive value of a 
history of depression, it is vital that women with a history of depression be counseled about the relationship between pregnancy and depression prior to conception.

\section{One method to incorporate depression screening \& counseling into preconception care}

Counseling about depression should become a routine part of preconception care. Health care providers who have contact with women during the preconception period can assist women in becoming aware of how pregnancy and depression can affect one another. By controlling depression prior to conception, just as one would with an illness such as diabetes or epilepsy, a woman's chances for healthy perinatal outcomes may improve [18].

The Wisconsin Association for Perinatal Care (WAPC) is a 35-year-old statewide, non-profit organization dedicated to the improvement of healthy outcomes for women, infants and their families. Since 1989, WAPC has been working toward infusing depression screening and counseling into preconception care by encouraging health care providers to ask women and their partners about their histories with depression and other mental illnesses prior to conception. One tool that has been developed by WAPC is an information sheet entitled Women with Depression that answers four basic questions: How does depression affect pregnancy? How does pregnancy affect depression? How could medications for depression affect the pregnancy? What can you do before pregnancy?

\section{Case examples}

The following are two case examples illustrating the impact of depression on pregnancy and the positive effects of being educated prior to a subsequent depressive episode. Although these women were not educated about perinatal depression prior to their first pregnancies, these examples illustrate the importance of depression counseling in the interconception period as well. These cases demonstrate that addressing the questions in Women with Depression can provide an anticipatory contextual framework on which to develop a plan of care.

\section{Case example \#1}

Sara began experiencing symptoms of depression six months after giving birth to her first child. She had no prior history of depression and had received no counseling prior to conception about the symptoms and presentation of perinatal depression. Sara waited approximately six months before seeking help, but encountered numerous barriers to accessing care. She did eventually get into treatment and attended 15 sessions of interpersonal therapy.
Through both her therapy and additional self-education, Sara became quite informed about the effects and consequences of postpartum depression. Approximately six months after giving birth to her second child, Sara began experiencing depression again. This time, Sara sought treatment within one week of the presentation of symptoms. In addition to being able to recognize the signs of depression earlier, as reported by both her and her husband, Sara also remarked on how much easier it was to access treatment, as she knew how to "navigate the system." She knew what type of therapy worked for her and was able to resolve her depression in only seven sessions.

\section{Case example \#2}

Melanie had a history of anxiety and depression prior to conception, but was never diagnosed or treated. Melanie experienced extreme continuous sleep disturbances almost immediately after the birth of her first child. When this problem had not resolved itself within two months, Melanie sought treatment. She was initially reticent about taking medications because she was breastfeeding her infant, but at nine months postpartum, Melanie began a regimen of paroxetine, which finally resolved the depression.

After Melanie's second birth, she felt slight anxiety about the possibility of suffering from postpartum depression again, but this time had a plan of action in place. When she began having trouble sleeping at two months postpartum, she immediately contacted her therapist and began treatment. She was once again hesitant about taking medication, but at nine months postpartum, began taking paroxetine on the recommendation of her care provider. Although she waited to begin medications with the second depressive episode, Melanie reported having "a kind of comfort knowing I was on paroxetine before and it didn't hurt my baby."

\section{Benefits of preconception counseling for women with a history of depression}

The benefits of counseling women about depression prior to conception are numerous. By treating depression as a chronic condition and by actively managing depression prior to conception, a woman's chances of being prepared for a depressive episode during the perinatal period greatly increase. In a recent meta-analysis of psychosocial and psychological interventions to reduce the risk of postpartum depression there was no statistically significant effect on the prevention of postpartum depression [19]. In the absence of preventive treatment, then, understanding postpartum depression could allow families to anticipate possible consequences of maternal depression so they could take steps to help assure healthy outcomes [20]. By fully disclosing the increased risk of de- 
pression if she chooses to become pregnant, a woman can make informed decisions about planning for a family [21]. If the woman is taking antidepressant medication, she can discuss the impact of those medications on her future pregnancy and breastfeeding. By working with her health care provider, steps can be taken to change the medication or dose if necessary.

One of the most devastating effects of perinatal depression is that it occurs at a time when most women feel they should be experiencing joy. By educating women prior to pregnancy or birth about the high prevalence of perinatal depression, they may be better prepared for an episode, should it occur. Women with a history of depression especially should have a plan of action in place in the event of perinatal depression.

Women who have suffered from depression once may be more likely to recognize the signs and symptoms of a subsequent episode whether or not the first episode occurred preconceptionally or postpartum. This may be especially true if they are counseled about the possibility of a subsequent episode occurring. Women are not the only ones who can be counseled about the interaction between pregnancy and depression. Loved ones often do not know how to help women suffering from perinatal depression and cannot understand why the woman is depressed. By counseling significant others and family members, they, too, can become better prepared and in addition, can help recognize signs of severe depression and assist with accessing treatment.

Seeking treatment may present fewer challenges to women who have been successfully treated and counseled about depression as well. Women with a history of depression can be counseled about seeking treatment at the earliest possible sign of depression, rather than waiting, as so often happens in primary episodes. The earlier treatment can be sought, the better the outcomes [22]. Stigma may be reduced as well if women are counseled ahead of time, making them more open to seeking treatment and more comfortable with their treatment decisions.

\section{Conclusions}

Testimonials from affected women suggest that counseling women with a history of depression leads to improved outcomes should they suffer from a subsequent episode during the perinatal period. Women may be more likely to recognize the signs of depression, more likely to seek treatment earlier and will know what treatment options work for them. Due to an earlier recognition of depression, treatment may have a shorter duration as well. In addition, by counseling women about depression prior to conception, they and their families will have time to develop a plan of action should a depressive episode occur and will be equipped to make informed decisions about family planning. Tools such as Women with Depression may be beneficial in both educating consumers and assisting providers with consultations about depression prior to pregnancy or during the interconception period.

The major challenge currently lies in convincing health care providers to address women's mental health and to screen and counsel for depression during the preconception period. Intervention strategies using medication or counseling have not been significantly effective in preventing postpartum depression. Anticipating the potential interplay between depression and pregnancy may be an intervention that can lead to better mental health and perinatal outcomes.

Future research should address the content and timing of preconception counseling, including what information is needed and when and how it should be disseminated. Treatment algorithms and strategies could then be developed for practitioners and families to help prepare for the postpartum period. Finally, well-controlled intervention studies utilizing the materials developed could focus on maternal mental health, perinatal, and infant outcomes to answer questions about efficacy and effectiveness.

\section{References}

1. Gaynes BN, et al. Perinatal depression: Prevalence, screening accuracy, and screening outcomes. Agency for Healthcare Research and Quality. Evidence Report/Technology Assessment. 2005 Number 119.

2. O'Hara JW, Swaim AM. Rates and risk of postpartum depression: a meta-analysis. Int Rev Psychiatry. 1996;8:37-54.

3. Hobfall SE, et al. Depression prevalence and incidence among inner-city pregnant and postpartum women. J Consult Clin Psychol. 1995;63:445-53.

4. Cox JL, Murray D, Chapman G. A controlled study of the onset, duration and prevalence of postnatal depression. Br J Psychiatry. 1993;163:27-31.

5. Murray L, et al. Self-exclusion from health care in women at high risk for postpartum depression. J Public Health Med. 2003;25(2):131-7.

6. Pajulo M, et al. Antenatal depression, substance dependency, and social support. J Affect Disord. 2001;65:9-17.

7. Beck CT. The effects of postpartum depression on maternal-infant interaction: a meta-analysis. Nurs Res. 1995;44:298-304.

8. Field T. Maternal depression effects on infants and early interventions. Prev Med. 1998;27:200-3.

9. Field $\mathrm{T}$, et al. Pregnancy anxiety and comorbid depression and anger: effects on the fetus and neonate. Depress Anxiety. 2003; $17: 140-51$

10. Rahman A, et al. Impact of maternal depression on infant nutritional status and illness: a cohort study. Arch Gen Psychiatry. 2004;61:946-52.

11. Paarlberg KM, et al. Psychosocial predictors of low birthweight: a prospective study. Br J Obstet Gynaecol. 1999;106:834-41.

12. Orr ST, James SA, Blackmore-Prince C. Maternal perinatal depressive symptoms and spontaneous preterm births among AfricanAmerican women in Baltimore, Maryland. Am J Epidemiol. 2002;156:797-802.

13. Wisconsin Association for Perinatal Care Screening for prenatal and postpartum depression. $2003 \mathrm{http}: / /$ www.perinatalweb.org/association/pub_pStateMoreBlues.html

14. Wisner KL, Parry BL, Piontek CM. Postpartum depression. N Eng J Med. 2002;347:194-9. 
15. Williamson V, McCutcheon H. Postnatal depression: a review of current literature. Aust J Midwifery. 2004;17:11-6.

16. Beck CT. Predictors of postpartum depression: an update. Nurs Res. 2001;50:275-85.

17. Altshuler LL, Hendrick V, Cohen LS. Course of mood and anxiety disorders during pregnancy and the postpartum period. J Clin Psychiatry. 1998;59(Supp 2):29-33.

18. Zimmer-Gembeck MJ, Helfand M. Low birthweight in a public prenatal care program: behavioral and psychosocial risk factors and psychosocial intervention. Soc Sci Med. 1996;43:187-97.
19. Dennis CL. Psychosocial and psychological interventions for prevention of postnatal depression: systematic review. BMJ. 2005;331:15-23.

20. D'Afflitti J. A psychiatric clinical nurse specialist as liaison to OB/GYN practice. J Obstet Gynecol Neonatal Nurs. 2005;34: 280-5.

21. Pariser SF, Nasrallah HA, Gardner DK. Postpartum mood disorders: clinical perspectives. J Womens Health. 1997;6:421-34.

22. Campagne DM. The obstetrician and depression during pregnancy. Eur J Obstet Gynecol Reprod Biol. 2004;116:125-30. 